\title{
THE WOLF-RAYET CONNECTION - LUMINOUS BLUE VARIABLES AND EVOLVED
} SUPERGIANTS

ROBERTA M. HUMPHREYS

University of Minnesota

Department of Astronomy

116 Church Street S.E.

Minneapolis, Minnesota 55455

\begin{abstract}
The physical characteristics and behavior of evolved massive stars in three different mass ranges are reviewed with application to whether they may eventually evolve to the WR stage 1 . $>40-50 \mathrm{M}_{\odot}$ as LBV's, 2. $\sim 30-40 \mathrm{M}_{\odot}$ as cool hypergiant-OH/IR stars and 3. $\sim 10-30 \mathrm{M}_{\odot}$ as red supergiant-OH/IR stars. I emphasize the importance of the relatively short but high mass loss phases as LBV's and as OH/IR stars in determining the fate of massive stars from 10 to $100 \mathrm{M}_{\odot}$.
\end{abstract}

\section{Introduction}

About ten years ago it was realized that the observed HR diagram has an upper luminosity limit. Humphreys and Davidson (1979) first drew attention to the empirical boundary and its physical significance for the evolution of massive stars. We proposed then that an instability causing rapid and unsteady mass loss was the basic explanation for the upper luminosity boundary. We emphasized the temperaturedependence of the boundary for the most luminous hot stars, the lack of cooler counterparts at similar luminosities and the temperatureindependent limit to the luminosities of the cool hypergiants. All of the stars that lie on or near this boundary are highly unstable and the highest mass loss rates are observed along this luminosity limit (de Jager 1984, 1988). The temperature-dependent boundary for the hot stars is marked by the presence of some very luminous unstable stars including such famous stars as $\eta$ Car, P Cyg, S Dor and the Hubble-Sandage variables in M31 and M33, now known collectively as the Luminous Blue Variables (LBV's). The temperature dependence for the hot stars suggests that this boundary defines a critical location in the HR diagram one that is mass dependent. This plus the lack of cooler counterparts, indicates that stars above some critical mass probably do not evolve to cooler temperatures. Based on observed HR diagrams in combination with evolutionary tracks, this critical mass is probably near 40-50 $\mathrm{M}_{\odot}$.

\section{Luminous Blue Variables - The Evolution of Stars $>40-50 \mathrm{M}_{\odot}$}

The LBV's are evolved, very luminous, unstable hot supergiants which 
suffer irregular ejections. The cause of their instability is very likely radiation pressure, resulting in a greatly enhanced average mass outflow ( $\sim 10^{-4} \mathrm{M}_{\odot} / \mathrm{yr}$ ) which leads to the formation of a pseudophotosphere (Leitherer et al. 1985) at visual maximum. At this stage the slowly expanding $(100-200 \mathrm{~km} / \mathrm{s})$ envelope is $\operatorname{cool}(8000-9000 \mathrm{~K})$ and dense $\left(\mathrm{N} \sim 10^{11} \mathrm{~cm}^{-3}\right)$, and the star resembles a very luminous $\mathrm{A}$ type supergiant. At minimum light, or the quiescent state, the LBV is at its "normal" high temperature ( $>15000-20000 \mathrm{~K})$ and the massloss rate is lower. During these variations the bolometric luminosity remains essentially constant. The visual light variations are caused by the apparent shift in the star's energy distribution driven by the instability. The transitions from the minimum to maximum state at constant bolometric luminosity are shown for the best studied LBV's on the accompanying HR diagram (Figure 1).

The close connection between LBV's and Of/WN9 stars has been known for some time and provides strong support for suggestions that LBV's are predecessors of WR stars. R127 in the LMC is perhaps the best example. Originally one of the few known of/WN9 stars first recognized by Walborn (1977), it recently erupted (Stahl et al. 1984). It is still at maximum light with a spectrum nearly identical to S Dor. AG Car at minimum light and HDE $269582=$ MWC112 also have spectra that very closely resemble the of/WN9 stars. Two other Of/WN9 stars in the LMC, R84 and R99, are also variable but with smaller amplitudes (Stahl et al. 1984) and are potential LBV's. Obviously this group of stars should be closely monitored for LBVlike behavior and we should try to identify their counterparts in our galaxy.

Most LBV's show some evidence for an excess of IR radiation and the presence of circumstellar ejecta (Humphreys et a1. 1984;

Leitherer et al. 1985; McGregor et al. 1988) produced by the high mass loss and ejection of shells from the LBV's. Some of this ejecta is clearly visible as in the famous homunculus of $\eta \mathrm{Car}$. In $\eta$ Car the dust is thick enough to obscure the star (Westphal and Neugebauer 1969). The presence of a ring nebula as in the case of AG Car (Thackeray 1977; Stahl 1987) is fairly common. Stahl (1987) described ring nebulae around AG Car, He3-519 a candidate LBV-Of/WN9 star, R127 and S61. Another example is the ring nebula RCW58 around the known WN8 star HD96548 (Chu 1982; Smith et al. 1988) which very closely resembles those around the LBV's. With its low expansion velocity of only $87 \mathrm{~km} / \mathrm{s}$, like the slow winds of LBV's, the nebula may be a "fossil" from the previous LBV stage. Smith et al. (1988) however concluded that the low velocity, the presence of dust and $\mathrm{N}$ and $\mathrm{He}$ enrichment in the nebula were evidence of a red supergiant progenitor, but they are more consistent with an LBV.

Quantitative analyses of the ejecta from LBV's and the atmospheres of LBV's and related stars such as the of/WN9's show that they are nitrogen and helium-rich ( $\eta$ Car, Davidson et al. 1982, 1986; $\eta$ Car, Allen et al. 1985; AG Car, Dufour and Mitra 1987 and reviews by Walborn 1988, 1989).

The actual amount of mass lost as an LBV is especially relevant to possible evolution to the WR stage. The duration of the LBV stage is often estimated at $\leq 10^{4}$ yrs (see Maeder 1989) by comparing their numbers with WR stars, but I want to caution that the numbers of LBV's is incomplete. Because their quiescent phase may be decades long we may have missed several. R127 and the recent announcement of 
R110 (Stahl et al. 1990) are excellent examples. In the LMC there are now 5 confirmed LBV's. I will also include R84 and R99 as candidate LBV's for a total of 7 compared with 115 WR stars. Then $\mathrm{N}(\mathrm{LBV}) / \mathrm{N}(\mathrm{WR})$ is $\approx 0.06$. With a total lifetime of $\approx 5 \times 10^{5}$ yrs (Maeder and Meynet 1987) for WR stars, this gives an LBV lifetime of about $\geq 25000$ yrs.

Lamers (1989) gave a thorough review of the various mass loss rates during the LBV cycle and therefore I will only give a brief summary below for typical LBV's.

1. minimum light: Ex. R71 $6 \times 10^{-7} \mathrm{M}_{\odot} / \mathrm{yr}$

2. Maximum light [Mass loss during the maximum light of the moderate variations ( 2 mag) of the typical LBV is about 10 to 100 times greater than during quiescence.]

AG Car $2-7 \times 10^{-5} \mathrm{M}_{\odot} / \mathrm{yr}$

R71 $2 \times 10^{-5}$

$S$ Dor $3 \times 10^{-5}$

R127 $6 \times 10^{-5}$

Based on the normal mass loss rates of LBV's between minimum and maximum light and assuming the star spends half the time in each phase, Lamers (1989) determined a time-average normal mass loss rate of $\approx 10^{-5} \mathrm{M}_{\odot} / \mathrm{yr}$.

However there is increasing evidence that the LBV's pass through a much more violent stage, that we call eruptions or explosions, during which the mass loss rate is much higher; discussed by both Lamers $(1986,1989)$ and by Humphreys (1989). Eta Car is of course the most famous example. During its $1840^{\prime}$ s outburst, $\eta$ Car probably lost 2-3 $\mathrm{M}_{\odot}$ and its current $\mathrm{M}$ is $10^{-4}$ to $10^{-3} \mathrm{M}_{\odot} / \mathrm{yr}$. More typical examples are P Cyg, AG Car and R127. In its current quiescent stage, $P$ Cyg's $M$ is $\sim 1.5 \times 10^{-5} M_{\odot} / y r$. If it is like other LBV's then $M$ during its outburst was 10-100 times greater or $10^{-4}$ to $10^{-3} \mathrm{M}_{\odot} / \mathrm{yr}$. Its extended $\mathrm{H} \alpha$ and NII emission measured by Leitherer and Zickgraf (1987) from previous ejections corresponds to continuous mass loss of $4 \times 10^{-4} \mathrm{M}_{\odot} / \mathrm{yr}$. From direct imaging of circumstellar shells around AG Car, R127 and others Stahl (1987) estimated their kinematic ages and masses and concluded that their average mass-loss rate must be $>10^{-4} \mathrm{M}_{\odot} / \mathrm{yr}$. We (Humphreys et al. 1989) have also shown that a shell of photoionized gas around AE And contains $>6 \times 10^{-3} \mathrm{M}_{\odot}$. If this is the material ejected during its last maximum, which lasted $\sim 20$ years, then the $M$ was $>3 \times 10^{-3} M_{\odot} / y r$ during the ejection event.

The mass loss rates during these more violent eruptions as measured from the circumstellar ejecta are summarized below:

\begin{tabular}{lcll} 
& $M$ & Duration & \multicolumn{1}{c}{ Age } \\
$\eta$ Car & $10^{-1}$ & 25 years & 150 years \\
P Cyg & $4 \times 10^{-4}:$ & 60 years & 400 years \\
AG Car & $2 \times 10^{-4}$ & mean rate over & $10^{4}$ \\
R 127 & $1.7 \times 10^{-4}$ & mean rate over & $1.7 \times 10^{4}$ years \\
AE And & $>3 \times 10^{-3}$ & 20 years & 80 years \\
R71 & $7 \times 10^{-5}$ & - & 400 years
\end{tabular}

The total mass lost during the lifetime of an LBV is of course uncertain because we don't know the frequency of these more violent eruptions. There is evidence from the proper motions of older ejected material around $\eta$ Car that it has undergone more than one eruption at intervals of several hundred years. Lamers gives a 
suggested time-averaged mass loss rate of $\sim 2 \times 10^{-4} \mathrm{M}_{\odot} / \mathrm{yr}$ over the LBV lifetime for the violent eruptions. Thus assuming that all LBV's pass through one or more violent eruptions, they very likely shed $>5$ $M_{\odot}$ during their 25,000 years. This is very close to the mass loss of 5-10 $\mathrm{M}_{\odot}$ that a 50-100 $\mathrm{M}_{\odot}$ star must shed after core $\mathrm{H}$-burning to become a WR star (based on the models of Maeder and Meynet 1987). The total mass shed as an LBV may be mass dependent, so that a star like $\eta$ Car may lose more total mass.

The characteristics of the LBV's; their instability and resulting high mass loss rates, circumstellar ejecta and atmospheres enriched in nitrogen and helium, their very obvious connection to the Of/WN9 stars, and of course their crucial location in the HR diagram all lead to the conclusion that LBV's are progenitors of WR stars. Or, depending on one's perspective, the WR stars are post-LBV's. Consequently for the most massive stars $\left(>40-50 \mathrm{M}_{\odot}\right)$ the following evolutionary scenario is now generally accepted:

$$
\text { MS O star } \rightarrow \text { of } \rightarrow \mathrm{LBV} \leftrightarrow \text { Of } / \mathrm{WN} \rightarrow \mathrm{WR} \rightarrow \mathrm{SN} \text { (Type Ib) }
$$

Based on the spatial distributions of WR stars and 0 stars Conti et al. (1983) suggested that most WR stars derive from star $>40$ $M_{\circ}$ and independently Humphreys, Nichols and Massey (1985) looking at their membership in associations and clusters showed that the lower limit to the initial masses of WR stars are $>30 \mathrm{M}_{\odot}$ with the majority $>50 \mathrm{M}_{\odot}$. Van der Hucht et al. (1988) concluded that WN stars derive from progenitors in the mass range 28-35 $\mathrm{M}_{\odot}$ and WC from 25-60 $\mathrm{M}_{\odot}$ stars. Thus the evolution of evolved stars near the upper luminosity limit is critical and some of these stars may lose sufficient mass to become WR stars.

\section{THE COOL HYPERGIANTS - THE LUMINOSITY/STABILITY LIMIT AND THE EVOLUTION OF STARS NEAR $40 \mathrm{M}_{\odot}$}

The upper limit to stellar luminosities is usually assumed to be set by the balance between the acceleration due to gravity and the radiation pressure gradient a la Eddington. However the observed luminosity boundary is composed of two components - the temperaturedependent boundary for hot stars and its turnover at the cool star upper limit. The classical Eddington limit due to electron scattering does not show the dependence on temperature for the hot stars. However as the temperature decreases below $30000 \mathrm{~K}$ the opacity increases due to ions of HI, FeII, et al. A modified or opacity-dependent Eddington limit which decreases with temperature has been proposed and discussed by sedveral investigators (Humphreys and Davidson 1984; Appenzeller 1986; Lamers 1986; Davidson 1987; Lamers and Fitzpatrick 1988). The opacities reach a maximum and the Eddington luminosity a minimum at $10000 \mathrm{~K}$. The modified Eddington limit will then turn up again in the 8000-10000 $\mathrm{K}$ temperature range in agreement with the observed turnover in the luminosity/stability limit. Stars below the corresponding critical mass could then evolve to the red supergiant region.

But the situation may be more complicated. First attempts to calculate the location of the modified Eddington limit on the HR diagram have not been entirely successful (Lamers and Fitzpatrick 1988). The F, G, K and $M$ hypergiants in our galaxy and other local 
group galaxies define the observed upper luminosity limit for the cooler stars and these stars are all highly unstable. De Jager $(1980,1984)$, has suggested that the instability in these stars is produced by a turbulent pressure gradient due to the dissipation of mechanical energy. In a series of papers, he and his collaborators have measured supersonic microturbulent motions in the atmospheres of many of these stars.

I think that the observed lumiosity/stability limit is a consequence of 1) radiation pressure, i.e., the modified Eddington limit, which dominates in the hot stars, and 2) the turbulent pressure gradient in the atmospheres of the cool hypergiants which sets an upper boundary to their luminosities independent of radiation pressure in the hot stars.

The instability in the atmospheres of the cool hypergiants is evidenced by their variability in light and in their spectra, by high mass rates (up to a few $\times 10^{-4} \mathrm{M}_{\odot} / \mathrm{yr}$ ), and the presence of extensive circumstellar dust shells around many. Many of the intermediate-type hypergiants show evidence for shell ejections. $\rho$ Cas (F8Ia) is especially well known for its shell episode (1946-47) in which it decreased $1.5 \mathrm{mag}$ and had the spectrum of an M star. HR8752 (G0G5Ia+) one of the most luminous hypergiants has shown considerable spectroscopic variation usually attributed to shell ejection. But neither $\rho$ Cas or HR8752 has the large IR excess due to circumstellar dust from high mass loss observed around many very luminous $G$ supergiants (Humphreys et al. 1971). HR5171a (G8Iat) has one of the largest $10 \mu$ silicate features observed in late-type supergiants. This star is especially interesting because it has been getting fainter and redder with time suggesting that the amount of dust and the obscuration are increasing perhaps due to continuous high mass loss or to unstable fluctuations in its outer atmosphere.

Variable A in M33 is perhaps the most enigmatic of all of the cool hypergiants (Humphreys et al. (1988). It was one of the original Hubble-Sandage variables but its behavior is bizarre even for them. In 1950 it was one of the visibly brightest stars in M33, with the spectrum of a very luminous $F$ supergiant. It then rapidly declined in brightness by $3.5 \mathrm{mag}$ becoming faint and red after slowly increasing in brightness during the previous 50 years. It is still faint and red and has the spectrum of an $M$ supergiant not an emission-line hot star! It also has a large infrared excess and is today as bright at $10 \mu$ as it was at its visual maximum in 1950 . Variable $A$ is a very luminous $\left(M_{B \circ 1} \approx-9.5 \mathrm{mag}\right.$ ), highly unstable $\left(2 \times 10^{-4} \mathrm{M}_{\odot} / \mathrm{yr}\right)$ star. Its present spectrum is probably produced in an expanded pseudo-photosphere and is shedding its mass in a highdensity, low-velocity wind.

The most luminous $M$ supergiants include such well known stars as $\mu$ Cep but in addition to the relatively normal red supergiants with circumstellar dust there are also the supergiant $\mathrm{OH} / \mathrm{IR}$ sources. They are likely the most evolved M supergiants; $M$ supergiants that have lost sufficient mass that their dust shells are now optically thick. The mass loss rates from the supergiant OH/IR sources may be very high $10^{-4}$ to $10^{-3} \mathrm{M}_{\odot} / \mathrm{yr}$. A few with optically thin shells are visibly bright, like VY CMa (M3-5eIa), VX Sgr (M4-8eI), and S Per (M4eIa). Two highly obscured, highly luminous late-type stars in the LMC (Elias, Frogel and Schwering 1986) discovered by IRAS presumably belong to this group; $\mathrm{OH}$ emission has just been detected from one 
(Wood et al. 1986). What will eventually become of these supergiant OH/IR sources? Could they evolve to Wolf-Rayet stars, analogous to their less massive counterparts which become the central stars of planetary nebulae?

IRC+10420 may be a good candidate for such a star in transition from red supergiant to $\mathrm{WR}$ star. IRC +10420 has the spectrum of a very luminous $F$ supergiant (F8Iat) plus a very large IR excess from a circumstellar dust shell (Humphreys et al. 1973). It is also one of the earliest (warmest) known OH/IR sources (Giguere et al. 1976). Recent $\mathrm{OH}$ observations show that the $1665 \mathrm{MHz}$ feature is weakening while the $1612 \mathrm{MHz}$ feature is growing (Lewis et al. 1986). This is what we would expect if the dust shell were dissipating.

Interestingly it has also been getting visually brighter (Gottleib and Liller 1978). If this trend continues a very plausible model for IRC+10420 will be a post $M$ supergiant-OH/IR star blowing off its cocoon of dust and gas as it evolves to the left to warmer temperatures on the HR diagram.

I have estimated the lifetime of the supergiant OH/IR stage from the numbers of known $M$ supergiants (122) and supergiant OH/IR sources within $3 \mathrm{kpc}$ of the sun (VX Sgr, VYCMa, NML Cyg and S Per. Also my colleague T.J. Jones (1990) estimates that there should be three $\mathrm{OH} / \mathrm{IR}$ supergiants within $3 \mathrm{kpc}$ of the sun from the statistics of the $\mathrm{OH}$ surveys. This is good agrement, and therefore assuming the red supergiant lifetime is $10^{6}$ yrs the OH/IR star stage should be 230,000 yrs. Similarly in the LMC there are 95, confirmed red supergiants brighter than the $A G B$ limit ( $M_{B o l} \approx-7.2$ mag) but only one detected OH/IR source. This is because of the limits of sensitivity of the radio telescopes not because they don' $t$ exist. There are two other excellent candidates, IRAS 05346-6949 and MG46, both very dusty, high luminosity red supergiants. With three candidate $\mathrm{OH} / \mathrm{IR}$ sources in the LMC, the estimated duration of this stage is again $\approx 30,000$ yrs.

Using these lifetimes for the OH/IR stage combined with mass loss rates for the red supergiant stage from the compilation by de Jager et al. (1988) and those observed for the OH/IR stage plus the lifetimes in various stages from the models by Maeder and Meynet (1988), I have estimated the amount of mass lost as an evolved supergiant for stars of mass 40,25 and $20 \mathrm{M}_{\odot}$. The numbers are summarized in Table 1 .

By the time the $40 \mathrm{M}_{\odot}$ star has passed through the region of the cool hypergiants for the second time it will have lost half of its initial mass. From the tables by Maeder and Meynet (1988) the relative surface abundance of hydrogen for a star with $\sim 20 \mathrm{M}_{\odot}$ remaining will be down to $\sim .2$. It will also be close to the onset of carbon fusion and the star could be well on its way to becoming a WR star. Adopting the evolutionary "funnel" for WR stars presented by Moffat (1989) at the colloquium on LBV's (see Figure 1), we see that the cool hypergiants might enter the WR sequence as late WC's. Could the remnant circumstellar dust shells of the OH/IR phase account for the dust shells around the late WC-type stars? The dust shells associated with the WC9 star are carbon-rich while the red supergiant-OH/IR stars have oxygen-rich circumstellar dust (Cohen et a1. 1975). Thus the WC9 dust shells cannot be the fossil remnants of a previous red supergiant stage. Furthermore, the WC dust shells are

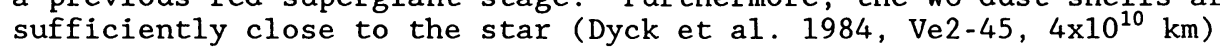




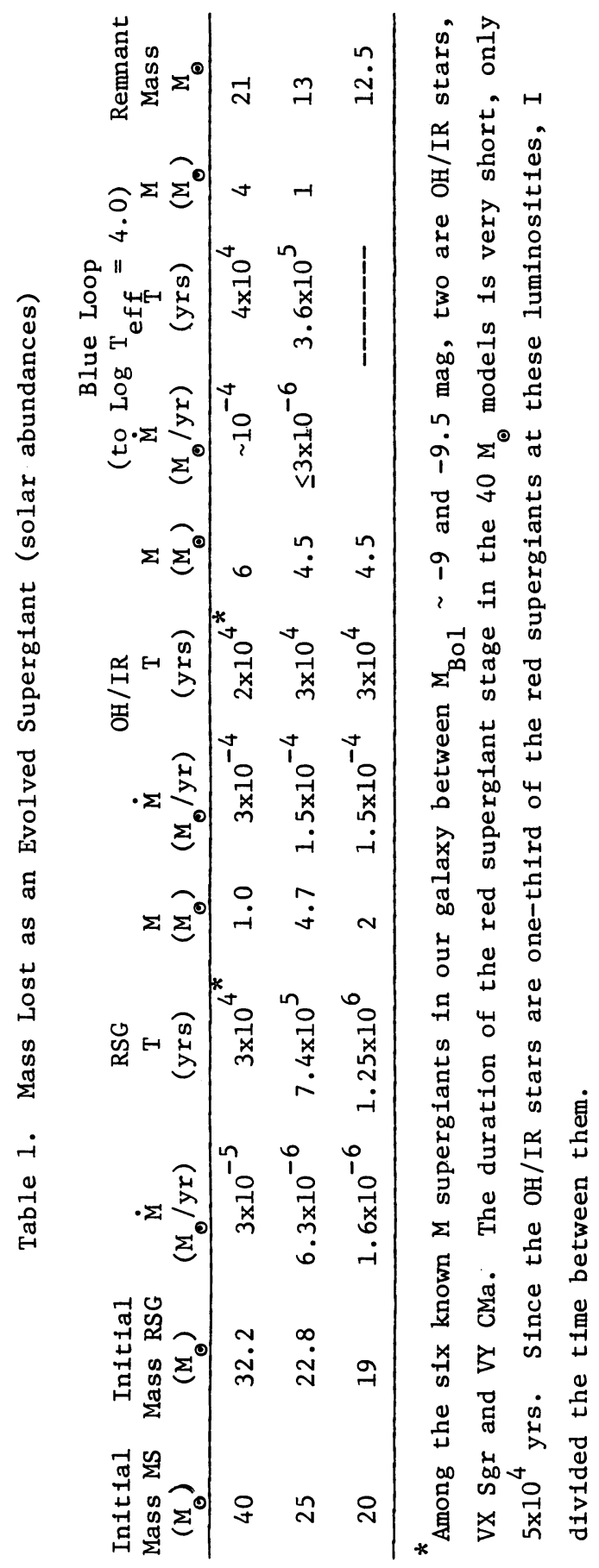




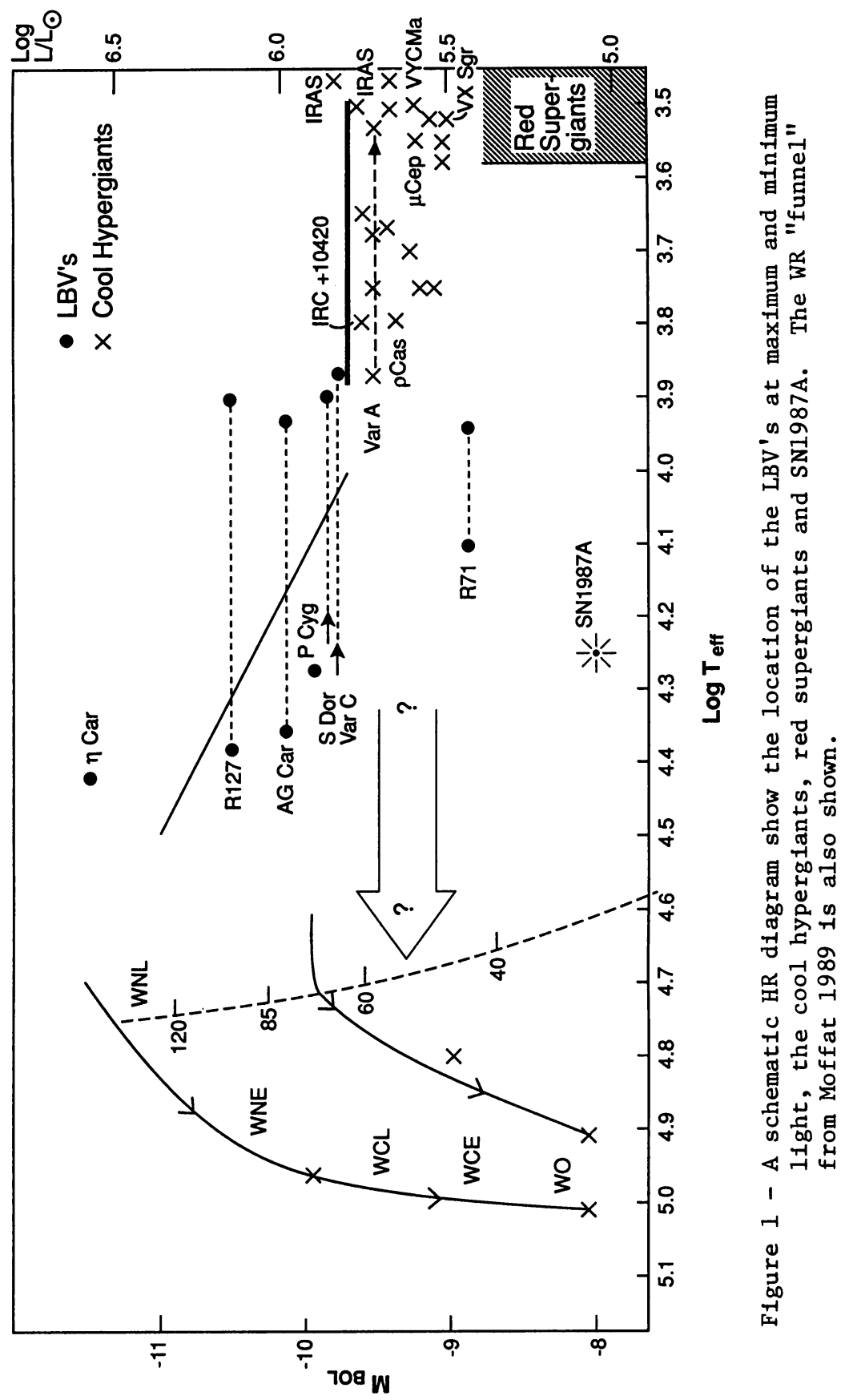


that the material must have been ejected recently. The WN stars are the more likely antecedents of red supergiant mass loss.

The results for the stars near the luminosity/stability limit are ambiguous but it is possible for some cool hypergiants to lose sufficient mass to become WR stars; although there is no direct connection with a particular subclass of WR star. However, this scenario depends critically on the properties and interpretation of one star, IRC+10420 as an evolved supergiant shedding its remaining hydrogen envelope. Thus for stars in the 30-40 $\mathrm{M}_{\odot}$ range, there are still two possibilities.

$$
\begin{aligned}
& \text { MS O star } \rightarrow \mathrm{BSG} \rightarrow \mathrm{YSG} \rightarrow \mathrm{RSG} \rightarrow \mathrm{OH} / \mathrm{IR} \rightarrow \mathrm{YSG} \rightarrow \mathrm{WR} \rightarrow \mathrm{SN}(\text { Type Ib) } \\
& \text { or RSG } \rightarrow \mathrm{OH} / \mathrm{IR} \rightarrow \mathrm{SN} \text { (Type II) }
\end{aligned}
$$

\section{EVOLVED SUPERGIANTS IN THE 15 TO $30 \mathrm{M}_{\odot}$ RANGE}

We know that the progenitors of many Type II supernovae must have very extended atmospheres or envelopes that are then illuminated by the supernova explosion. The most likely candidates are the red supergiants and they are generally adopted in the models for the Type II supernovae (Woosley and Weaver 1986). SN1987A and its progenitor $\mathrm{Sk}-69^{\circ} 202$ showed us that some red supergiants in this somewhat lower mass range evolve back to the blue and explode as supernovae as a hot more compact supergiant.

Do some evolved supergiants of lower initial masses also reach the WR stage before becoming supernovae? The numbers in Table 1 show that stars in this mass range do not shed their entire hydrogen envelopes in the RSG-OH/IR stage. Again referring to the tables by Maeder and Meynet, by the time stars of initial masses of 25 and 20 $M_{\circ}$ are down to 13 and $12 M_{\circ}$ respectively, their hydrogen surface abundances would still be $\approx .4$, far from the bare hydrogen-deficient core of the WR star. An additional high mass loss phase would still be required. The two low-luminosity relatively cool LBV's, R71 and R110 might qualify as post RSG's for stars in the 25-30 $\mathrm{M}_{\odot}$ initial mass range, and would be an additional stage where high mass loss could occur. What would be the cause of an instability at this stage in the star's evolution?

We know that $\mathrm{Sk}-69^{\circ} 202$, a B3 supergiant, had previously been a red supergiant from the detection of its circumstellar shell of gas and dust (Frannson et al. 1988; Wampler and Richicci 1988) which was e jected about 10,000 yrs. before. According to Woosley (1988) and others, $6 M_{\odot}$ was left in the helium core of $\mathrm{Sk}-69^{\circ} 202$, and its hydrogen envelope still had 5-10 $\mathrm{M}_{\odot}$. Thus at the time of the explosion $\mathrm{Sk}-69^{\circ} 202$ still had $11-16 \mathrm{M}_{\odot}$ remaining from its initial mass of 18-20 $\mathrm{M}_{\odot}$. The numbers in Table 1 are within this range, although on the low end; however the mass loss rates could be lower for the LMC red supergiants.

The poster at this meeting by Rolf Kudritzki, Hans Groth and myself describes a group of A-type supergiants in the Magellanic Clouds with anomalously strong hydrogen lines and U-B colors that are too red. On the HR diagram, these stars are predominantly between $M_{B o 1}=-6$ and -8 mag corresponding to initial masses of $10-20 M_{\odot}$. We show that the hydrogen line profiles can be reproduced by an enhanced 
helium abundance, of the order of .5 by number. This may seem rather high, but although the Maefer and Meynet tracks for 15 and $20 \mathrm{M}_{\odot}$ do not evolve back to the blue after the red supergiant stage, the 25 and $40 \mathrm{M}_{\odot}$ tracks show relatively high He abundances at the surface during the blue loop in this temperture range. Spectroscopically normal A-type supergiants are also found in the same luminosity/ temperature region of the HR diagram as these peculiar stars in both galaxies. Thus we are suggesting that these stars are post red supergiants and are excellent candidates for the type of star that eventually exploded as SN1987A.

It is interesting that none of these stars are found brighter than $M_{B O 1} \approx-8 \mathrm{mag}$. This stage is either very short or perhaps it doesn't exist for stars much more massive than $20 \mathrm{M}_{\odot}$. It is also important to determine whether these stars exist in our galaxy or whether this blue loop at these masses is a characteristic of lower metallicity systems.

\section{FINAL REMARKS}

During the past decade most of our interest in massive star evolution and our greatest uncertainties have focussed on the uppermost part of the HR diagram and the evolution of the most massive stars. Although there is still a lot of physics to be learned about the origin of the luminosity/stability limit, I think the greatest uncertainties now concern the final stages in the 10-30 $\mathrm{M}_{\odot}$.

Some possibilities include:

$$
\begin{aligned}
0 \text { or B } & \text { star } \mathrm{MS} \rightarrow \mathrm{BSG} \rightarrow \mathrm{RSG} \rightarrow \mathrm{OH} / \mathrm{IR} \rightarrow \mathrm{SN} \text { (Type II) } \\
& \text { or RSG }(\mathrm{OH} / \mathrm{IR}) \rightarrow \mathrm{YSG} \rightarrow \mathrm{BSG} \rightarrow \mathrm{SN} \text { (Type II) } \\
& \text { or RSG } \rightarrow \mathrm{YSG} \rightarrow \mathrm{BSG} \rightarrow \mathrm{RSG} \rightarrow \mathrm{OH} / \mathrm{IR} \rightarrow \mathrm{SN} \text { (Type II) }
\end{aligned}
$$

In this brief review, I have tried to emphasize the importance of the relatively short but high mass loss phases of the LBV's and $\mathrm{OH} / \mathrm{IR}$ supergiants in determining the fate of stars from 10 to $100 \mathrm{M}_{\odot}$.

\section{References}

Allen, D.A., Jones, T.J. and Hyland, A.R. (1985), Astrophys. J. 291, 280.

Appenzeller, I. (1986), IAU Symposium 非16, Luminous Stars and Associations in Galaxies, pg. 139.

Chu, Y.-H. (1982), Astrophys. J. 254, 578.

Cohen, M., Barlow, M.J., and Kuhi, L.V. (1975), Astron. Astrophys. $40,291$.

Conti, P.S., Garmany, C.D., de Loore, C. and Vanbeveren, D. (1983), Astrophys. J. 274, 302 .

Davidson, K. (1987), Astrophys. J. 317, 760.

Davidson, K., Dufour, R.J., Walborn, N.R. and Gull, T.R. (1986), Astrophys. J. 305, 867.

Davidson, K., Walborn, N.R. and Gull, T.R. (1982), Astrophys. J. (Letters) 254, L47.

de Jager, C. (1980), The Brightest Stars (Reidel: Dordrecht). 
de Jager, C. (1984), Astron. Astrophys. 138, 246.

de Jager, C., Nieuwenhuijsen, H. and van der Hucht, K. (1988), Astron. Astrophys. 193, 375.

Dufour, R.J. and Mitra, P. (1987), Bul1. A.A.S. 19, 1020 .

Dyck, H.M., Simon, T. and Wolstencroft, R.D. (1984), Astrophys. J. 277,675 .

Elias, J.H., Frogel, J.A. and Schwering, P.R.W. (1986), Astrophys. J. 302,675 .

Fransson, C., Cassatella, A. Gilmozzi, R., Kushner, R.P., Panagia, N. , Sonneborn, G. and Wamsteker, W. (1989), Astrophys. J. 336, 429.

Giguere, P.T., Woolf, N.J. and Webber, J.C. (1976), Astrophys. J. (Letters) 207, L195.

Gottleib, E.W. and Liller, W. (1978), Astrophys. J. 225, 488 .

Humphreys, R.M. (1989), IAU Colloquium 非13, Physics of Lumious Blue Variables, p. 3 .

Humphreys, R.M., Blaha, C., D'Odorico, S., Gull, T.R. and Benvenuti, P. (1984), Astrophys. J. 278, 124.

Humphreys, R.M. and Davidson, K. (1979), Astrophys. J. 232, 409.

Humphreys, R.M. and Davidson, K. (1984), Science 223, 243 .

Humphreys, R.M. and Davidson, K., Stahl, 0., Wolf, B. and Zickgraf, F.-J. (1989), IAU Colloquium 非13, Physics of Luminous Blue Variables, p. 303 .

Humphreys, R.M., Jones, T.J. and Gehrz, R.D. (1988), Astron. J. 94, 315 .

Humphreys, R.M., Strecker, D.W., Murdock, T.L. and Low, F.J. (1973), Astrophys. J. (Letters) 179, L49.

Humphreys, R.M., Strecker, D.W. and Ney, E.P. (1971), Astrophys. J. (Letters) 167, L35.

Jones, T.J. (1990), private communication.

Lamers, H.J.G.L.M. (1986), IAU Symposium 非16, Luminous Stars and Associations in Galaxies, p. 157.

Lamers, H.J.G.L.M. (1987), in Workshop on Instabilities in Luminous Early-Type Stars, p. 99.

Lamers, H.J.G.L.M. (1989), IAU Colloquium 非13, Physics of Luminous Blue Variables, p. 135 .

Lamers, H.J.G.L.M. and Fitzpatrick, E. (1988), Astrophys. J. 324, 279 .

Leitherer, C., Appenzeller, I., Klare, G., Lamers, H.J.G.L.M., Stahl, 0., Waters, L.B.F.M. and Wolf, B. (1985), Astron. Astrophys. 120, 113 .

Leitherer, C. and Zickgraf, F.-J. (1987), Astron. Astrophys. 174, 103.

Lewis, B.M., Terzian, Y. and Eder, J. (1986), Astrophys. J. (Letters) $302, \mathrm{~L} 23$.

Maeder, A. (1989), IAU Colloquium 非13, Physics of Luminous Blue Variables, p. 15 .

Maeder, A. and Meynet, G. (1987), Astron. Astrophys. 182, 243.

Maeder, A. and Meynet, G. (1988), Astron. Astrophys. Suppl. 76, 411.

Moffat, A.F.J. (1989), IAU Colloquium 非13, Physics of Luminous Blue Variables, p. 229.

McGregor, P.J., Hyland, A.R. and Hillier, D.J. (1988), Astrophys. J. 324, 1071 .

Smith, L.J., Pettine, M., Dyson, J.E. and Hartquist, T.W. (1988), Mon. Not. Roy. Astron. Soc. 234, 625. 
Stahl, O. (1987), Astron. Astrophys. 182, 229.

Stahl, O., Wolf, B., Leitherer, C., Zickgraf, F.-J., Krautter, J. and de Groot, M. (1984), Astron. Astrophys. 140, 459 .

Stahl, 0., Wolf, B., Klare, G., Juttner, A. and Cassatella, A., Astron. Astrophys., 228, 379.

Thackeray, A.D. (1977), Mon. Not. Roy. Astron. Soc. 180, 95.

van der Hucht, K.A., Hidayat, B., Admiranto, A.G., Supelli, K.R. and Doom, C. (1988), Astron. Astrophys. 199, 217.

Walborn, N.R. (1977), Astrophys. J. 215, 53.

Walborn, N.R. (1988), IAU Colloquium 1 108, Atmospheric Diagnostics of Stellar Evolution, p. 70.

Walborn, N.R. (1989), IAU Colloquium 非13, Physics of Luminous Blue Variables, p. 27.

Wampler, J. and Richicci, A. (1988), ESO Messenger 52, 14. Wood, P.R., Bessel1, M.S. and Whiteoak, J.B. (1986), Astrophys. J.
(Letters) 306, L81.

Woosley, S.E. (1988), Astrophys. J. 330, 218.

Woosley, S.E. and Weaver, T.A. (1986), Ann. Rev. Astron. Astrophys. $24,205$.

\section{DISCUSSION}

Leung: Earlier in my paper I brought up two contact systems with combined masses, of over 50 , or about $100 M_{\odot}$. They suggest that in double stars evolution could preserve some of their, say, wind lost. Presumably, there are large amounts of material surrounding the components. The IUE spectra are extremely complicated and do not look like ordinary $\mathrm{K}$ supergiants at all. As a matter of fact, they look very similar to U Gem stars with most of the characteristic strong emission lines. These systems have periods of about 100 days or longer.

Chu: If LBV's turn into WN's, why is oxygen severely depleted in LBV ejecta ( $\eta$ Car and AG Car) but not depleted much at all in ejecta around WN stars?

Humphreys: Oxygen is not overabundant in LBV ejecta. We think their $N$ and $H e$ enrichment is the result of $C N O$ process. If WN's are more evolved they may be closer to onset of $C$-fusion. It would be appropriate for the interior people to consider this problem.

Maeder: Allow me to make a general constructive comment on observations about evolutionary connections. Ideally, one should use (1) location in the HRD, (2) surface composition, (3) pulsations. In that respect, I emphasize that the periods are not expected to be the same for stars on bluewards or redwards tracks. Maybe in some years observations will lead to such multiple comparisons, which would be very constraining.

Humphreys: I did not discuss the periods of variability. I merely mentioned that the cool hypergiants are variable in light as additional evidence of their instability. Most of the cool hypergiants show irregular light variations. 
Cherepashchuk: I would like to say something about the progenitors of WR binaries. According to our interpretation (1988), the massive $\mathrm{O}+\mathrm{O}$ binary system RY Scuti may be considered as the progenitor of the WR+O binary, because the more massive component in this system is surrounded by a thick accretion disk and the less massive companion has weak emission of $\mathrm{HeII} 4686$ and is an intensively mass loosing star, becoming a WR star. On the other hand, the SS433 object may be considered as the progenitor of the WR+c binary system, because, according to our light curve solution for this eclipsing binary system (Cherepashchuk, 1988), the normal star $\left(M \approx 20 M_{\odot}\right)$ is filling its Roche lobe and is loosing mass with a high rate on thermal time scale: $\dot{M} \approx 10^{-4} M_{\odot} \cdot y r^{-1}$. So our results confirme the suggestion made by van den Heuvel (1981) that SS433 is a massive binary observed at a second mass exchange phase of evolution and that the normal star in SS433 is evolutionary linked with the WR phase.

Humphreys: My remarks about massive stars only address single star evolution. The situation for initial masses could be very different in binaries and undoubtedly is.

Langer: RSG's, as any massive star, cannot directly evolve into WC stars, i.e., skip the WN stage, simply because $H e$-burning convective cores of massive stars are always smaller (in mass) than the $H$-burning convective cores.

Humphreys: I agree that the RSG's do not become WC's. If they become WR stars, they most likely become WN's. As yet, there is no direct connection as in the same sense as for the LBV's.

Massey: If Gallagher were here, he might bring up the issue of where the Hubble-Sandage variables in M33 are found. Are the known LBV's in M33 located in the same places as WR stars, or are they preferentially located in the inner-arm regions?

Humphreys: The Hubble-Sandage variables in M33 including Var A are in associations, in the spiral arms and in star forming regions. However, they are not in the large $H I I$ regions.

Owocki: In our attempt to understand the physical cause of the LBV phenomenon, I think it helpful to focus on the time scales. Outburst time scale of every few years is far longer than the dynamical time (e.g., sound travel time), and this rules out mechanisms like line-driven instability as well as interior perturbation. On the other hand, this is much shorter than an evolutionary time scale. Thinking of intermediate scales, perhaps we should consider the cooling time scale of the star's outer envelope or deep atmosphere.

Humphreys: In 1979 we suggested that it was interior evolution that brought the star to the stability limits resulting in some large eruption. The subsequent moderate variations $(\sim 2 \mathrm{mag})$ may be the star's attempt to continue to adjust its atmosphere to remain stable as interior evolution again pushes it to the stability limit.

Cassinelli: Is it true that the LBV's are up in the same general part of the HRD as the B [e] stars?

Humphreys: The B[e] supergiants do not exactly overlap with the LBV's on the HRD. They actually tend to occur seemingly systematically less luminous than the LBV's. They are not variable in light and the evidence of Zickgraf's work is, that they very likely have equatorial disks around. LBV's may also have equatorial disks, but we do not have the strong evidence as he does. He has the dual component model for the emission lines, where some of the emission is coming from the equatorial disk producing the very narrow lines while the broader emission lines that he sees in the same spectrum are coming from a polar region, perhaps. B[e] supergiants are not LBV's. My own suspicion is that the latter will proven to be binary systems. 


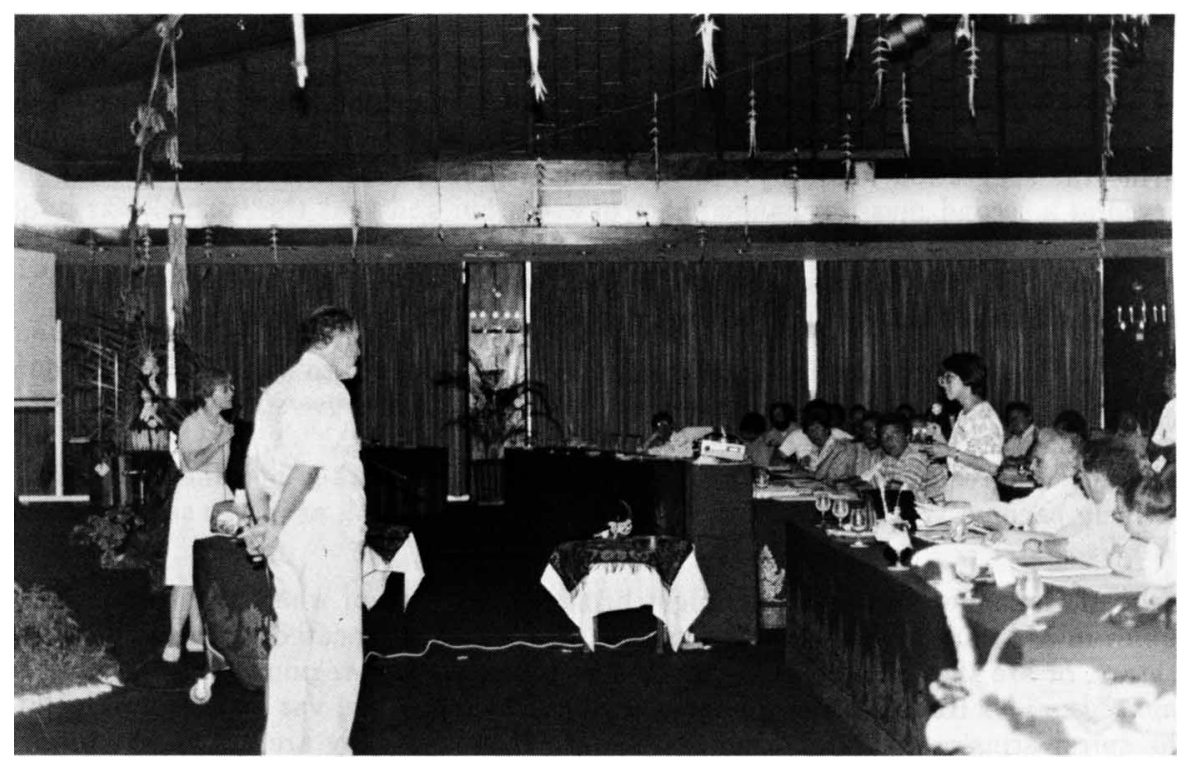

Roberta Humphreys, Peter Conti, You-Hua Chu 